\title{
Geochemical barriers as structural components of the geochemical systems evolution
}

\author{
Aleksandr Khaustov $^{1, *}$, and Margarita Redina ${ }^{1}$ \\ ${ }^{1}$ Peoples' Friendship University of Russia, Russia
}

\begin{abstract}
The term "geochemical barrier" (GCB) has been widely used in the Russian geochemical literature as a key concept of the distribution of elements and substances theory (incl. pollutions)although in the world research practice this term is not particularly represented. The assessment of the functional role of the geochemical barriers in relation to the properties and evolution of the geochemical systems (GCS)is demonstrated.The foundations of Haken synergy, the foundations of selforganization of systems and non-equilibrium (non-linear) thermodynamics of I. Prigogine and his school are used as a methodological framework. From the authors' point of view, GCB are considered as self-organizing components of GCS, in which physical and chemical processes are activated, leading to the transformation of atomic and molecular structures, chemical associations and individual chemical elements under the impact of active media (processes). They can be the defining phenomenon of the emergence and evolution of GCS. The concept of geochemical barriers is the foundation for technologies that are actively implemented for cleaning and protecting soils, groundwater and surface water, and the geological environment in general.
\end{abstract}

\section{Introduction}

GCBs are the most important components of GCS. The definition of the term "barrier" as applied to rocks, soils and landscapes, their typology, functional features and ecological significance are formulated by the Russian scholar A.I. Perelman and are presented in most detail in [1]. Thus, in relation to soils and landscapes, the GCB is " $\ldots$ an obstacle in the path of the movement of soil solutions and suspended solid particles of various sizes - mud, silty, sandy." According to the Great Encyclopedic Dictionary, GCB are the zones of a sharp decrease in the migration ability of any chemical elements, and this process is accompanied by their sedimentation from the solution and leads to their increased concentration, including industrial ones.

The research objective is to formulate an idea about GCB and their role in the formation of GCS from the standpoint of synergy and to demonstrate the processes occurring on GCB with the help of marker substances.

The spatial and temporal limitations (zonality) introduce a major uncertainty in the allocation of GCS taking into account the barriers. If they are not allocated (and the

* Corresponding author: akhaustov@yandex.ru 
selection is permissible with the help of borders that are mobile in natural systems in time and space), then GCB cannot existin principle, as they are secondary to zones. Virtually all the natural boundaries in systems are diffuse and created for the exchange of various substances, without which open systems cannot exist.

In the "Mountain Encyclopedia", the GCB are defined as "areas of the earth's crust where, in the direction of migration of chemical elements, one stable geochemical situation at a relatively short distance is replaced by another. At the same time, there is a decrease in the migration capacity of individual elements and their selective accumulation, up to the formation of ore bodies". There exist certain disadvantages from a semantic position. The concept of the geochemical situation stability in the direction of migration is not interpreted in principle. Migration is a non-stationary process; therefore, the concept of resistance is only theoretically applicable to it. A change of scenery is an indispensable condition for the existence of GCB. But GCB can occur without changing of geochemical situation and vice versa. It is all about the critical conditions for the interaction of factors and conditions for the migration of solutions. The main factor is the synergy of these processes. Essential in this definition is the selective nature of chemical elements in relation to barriers. Here we are very close to the function of borders.

From the standpoint of the analysis performed, the term "barrier" is not very accurate. Therefore, A.I. Opekunov (2012) proposed a classification of "borders - sections" and "borders - barriers" with different aggregation states, at which mass transfer is maximized due to the gradient of physicochemical parameters at the phase division. But parameter gradients are secondary to barriers and can exist without them. From the standpoint of geology and landscape science, the border area is an active framework phenomenon, an area of separation as well as interaction. This means that the functions of boundaries are not only prohibitive, but also permissive. The borders on the path of the energy masses for some of their types will be impenetrable, for others they are open. It is also important that emergence of the border or its identification formally turns a continuous (homogeneous) environment into a discrete one.

In the case of the influence of multidirectional factors that are approximately equal in their energy, resonant self-oscillatory structures are created; this leads to the destruction of boundaries and, therefore, systems. At the same time, autowave processes with a certain combination of oriented factors are the cause of inhomogeneities and, therefore, borders (resonant self-development according to A. Andronov).

Thus, the formation of HCB as a soft system can be demonstrated by the case of the formation of sedimentary rocks. Until a certain stage, these barriers can be washed away or pass through only certain amounts of substance depending on the strength of selfoscillations (wave-like variations of the barrier capacity). Such variations create surface and ocean flows, and the larger they are, the more diverse are the types of rocks formed (e.g. sandstones).

If the internal interaction exceeds the external one, the system tends to self-organize around certain centers (attractors). Its states before and after changing parameters are topologically identical (equivalent). Rough systems are filled with open areas in the functional space of all dynamic systems. Outside these areas (in particular, on their borders) there are non-rigid ("soft") systems. The delivery of matter and energy through the border is accompanied by bifurcation a change in the structure of the dynamic system due to the confrontation between the transfer processes (they disturb the equilibrium) and the internal relaxation processes (they tend to restore the equilibrium). Thus, a geochemical system cannot exist without geochemical barrier. Moreover, GCB can have prior relation to the formation of complete systems.

Thus, the "water-rock" interaction forms new minerals and, therefore, new properties of GCS[2-4]. Therefore, the GCB is an "active medium" capable of a higher level of space-time self-organization compared to "rough systems". So, we need fundamentally new approaches 
to studying them. A self-organizing GCS can take on conventionally fuzzy forms of borders for certain periods due to disequilibrium. In this sense, we move from spatial borders to time constraints. Most likely, micro-GCB are "born" and "die" on GCB to create more stable forms or structures - this is another function of GCB.

Given this goal, the research is aimed at the identification of chemicals or their atomicmolecular bonds (AMS) of mass flows, representative to identify evolutionary pathways of systems development and interpretation of the processes leading to this development. The most effective studies are carried out at the points of bifurcation of the trajectory of systems or at the geochemical borders-sections (phase transitions).

\section{Objective of study (formation and GCBstructure)}

The objective of this research is a GCB. Taking into account the above shown gaps in descriptions of barriers, we suggest a new definition: GCB is a dynamic element of GCS, which regulates the flow of energy and matter portions; borders (barriers) are a necessary structural element of open self-organizing systems. Natural systems are open and have negative feedback, leading to autowave processes. From these positions, GCB can be represented as a derivative of structural-phase transitions of two or more media. The simplest scheme of interaction between a relatively stable system (target system) and an active medium is presented on fig. 1.

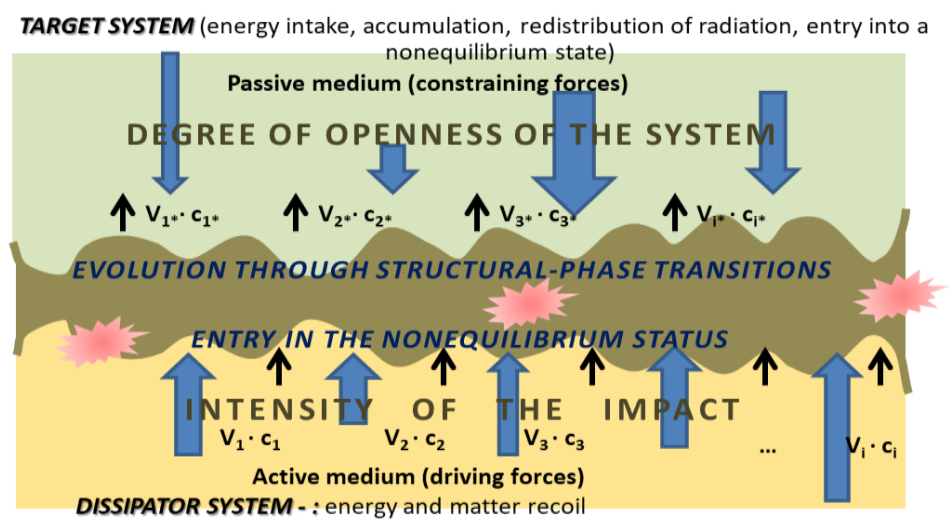

Fig. 1.Theoretical model of the occurrence of self-oscillations in the interaction of active and passive environments [5]

From the point of view of geochemistry, these are geochemical borders where the transformation, accumulation or conservation (possibly also dispersion) of flows of substances occurs. Thus, borders (barriers) are a necessary structural element of selforganizing GCS. The change in the structure or order is impossible without the differentiation of the structure of the elements. The variety of resonance forms of stable bonds in the system leads to the instability of the borders. This, paradoxical at first glance, judgment leads to a fundamental revision of the concept of GCB. Moreover, the borders determine the nonlinearity of the processes occurring in the system. As a rule, the heterogeneities are located near the borders of the system existence. Therefore the evolution of systems can occur from the border areas or their boundary states [6].

As example: the fractionation in the system "hydrocarbons-water-rocks" is that mainly high-molecular compounds (resins, asphaltenes, etc.) are accumulated in rocks; such compounds as benzene, toluene, xylene, etc., which have a relatively high solubility,permeate into aquifers. The solubility of hydrocarbons in water depends on their 
structure and molecular weight. Other things being equal, the lowest homologues have the highest solubility. Solubility among hydrocarbons with the same number of C-atoms falls in the series arenes - naphthenes (cyclic HC) - alkanes. Due to the increased concentration of arenes in water, the rate of their degradation becomes comparable with the rate of destruction of other, more digestible by microorganisms HC. This increases the water content of phenol, cresols and xylenols.

From modern positions GCB are considered as self-organizing components of GCS, in which activation of physical and chemical processes occurs, leading to the transformation of atomic and molecular structures and associations of chemical substances and individual chemical elements under the action of active media (processes) [7].

Thus the evolution of the system can occur from the border areas or border states. A schematic diagram of this evolution of GCS is presented in fig. 2.

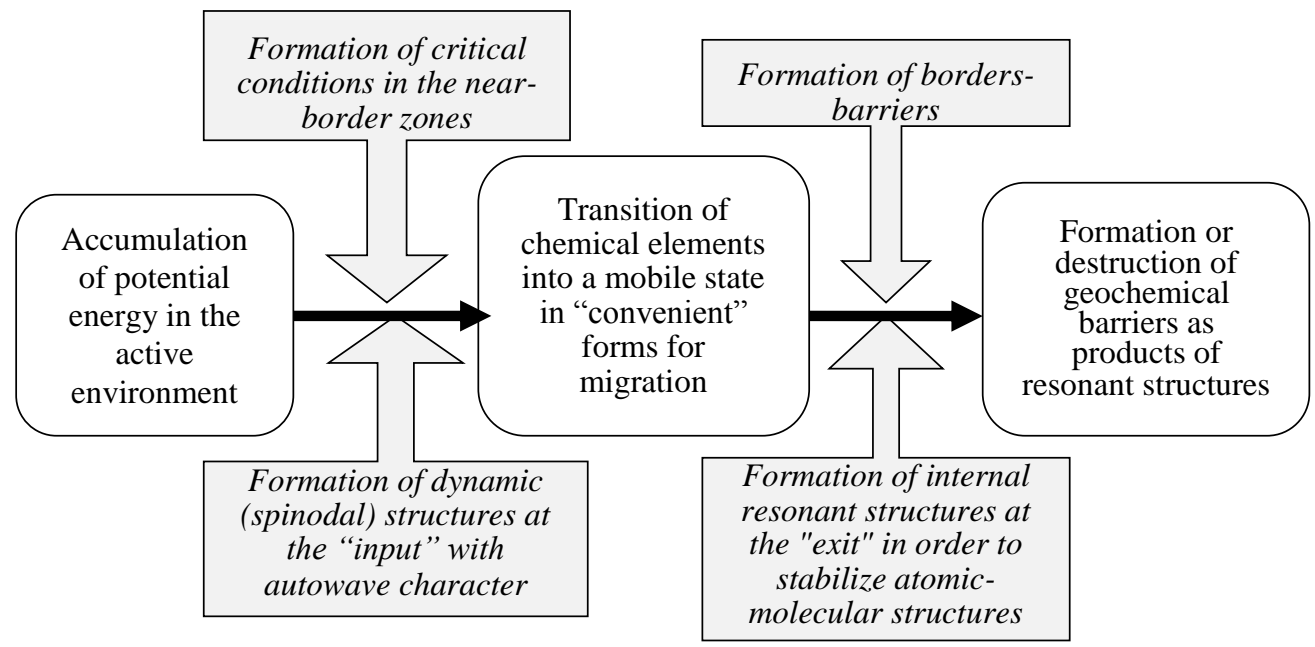

Fig. 2. Theoretical model of the sequence of physical and chemical processes of GCB formation: the general sequence of physical and chemical processes of barrier formation.

Consider the case of some pollutants entering a GCS. The more complex are the atomic and molecular structures, the less intensive are the processes of natural decomposition of oil and petroleum products. So, highly soluble alkanes degrade primarily, and cycloalkanes or naphthenes, which form up to $60 \%$ of the base oil, are very resistant to external influences.

Self-organization of systems requires an energy flow at the input of the system exceeding that at the output (the main condition of dissipation). The entropy component is generated from the total energy flow, that is, the energy produced by the system itself, which is necessary to support the functioning of self-organizing structures. Thus, in the formation of bottom sediments, the input energy flow is formed from all forms of energy (mechanical, light energy, thermal energy of the substance flow); the output flow is formed of the transformation energy of clay material and organic matter of sediments. This is how the bifurcation of the channel and the damping of the flow energy, for example, the formation of a marginal filter occurs $[8,9]$. The entropy component is generated from the total energy flow, that is, the energy produced by the system itself, which is necessary to support the functioning of self-organizing structures. The degradation of many pollutants into GCB and the artificial purification of the hydrosphere follow a similar pattern. 


\section{Conclusion}

The dominant role in the formation of space-time fluctuations of the system is created by GCB - an integral structural element for its self-maintenance (structuring). They give impetus to the redistribution of energy and matter in GCS and, consequently, their evolution. At the same time, rough systems can reduce the role of barriers through internal self-development.

Natural GCB is a product of spontaneous self-structuring and differentiation of the substance, its fluctuations, which is based on synergetic effects.

In destructive (catabolic) processes on GCB, autowave processes are caused by the change of biochemical and physicochemical processes with the dominant role of hydrodynamic moisture flows. The maximum intensity of destruction of chemical substances is achieved by hydration effects in the upper part of the geological section.

Consideration of "living matter" as a part of the geochemical system (living matter dissipative structure and active component) significantly changes our understanding of nonequilibrium geochemical systems at all levels of their organization.

The variety of components of the GCS determines its resistance to external influences.

Assessments of entropy confirm the decisive role of living matter not only for the formation of surface forms in the hydrocarbon genesis of pollution, but also for the formation of geochemical zonality during the migration of hydrocarbons from the surface to the aeration zone.

\section{References}

1. A. Perel'man, Vestnik Moskovskogo Universiteta. Ser.5 Geografiya, 4, 10-16 (1995)

2. S. Shvartsev, Bull. of the Rus. Academy of Sciences, 85 (5), 63-73 (2015)

3. S. Shvartsev, Bulletin of the Russian Academy of Sciences, 84(7), 618-628(2014)

4. I. Chenikov, Khimiya i fizika nefti, Chemistry and physics of petroleum. Krasnodar: Kuban State Technological University Publ., 293 (2010)

5. A. Khaustov, O. Korobova, M. Redina, International multidisciplinary scientific geoconference SGEM, 18, 869-874 (2018)

6. A. Khaustov, Geoekologiya. Inzhenernaya geologiya, gidrogeologiya, geokriologiya, 6, 1-11 (2006)

7. A. Khaustov, Geochemistry of landscapes (to A.I. Perelman's 100 anniversary). Moscow: Geographical Faculty of the MSU Publ. 64-67 (2016)

8. A. Lisitzin, Biogeochemical cycling and sediment ecology. Springer, Dordrecht, 69-103 (1999)

9. I. Nemirovskaya, A. Khaustov, M. Redina. Izvestiya Rossiyskoy akademii nauk. Seriya geograficheskaya, 6, 49-56(2018) 